\title{
Modelling on Dynamic Spectrum Planning of Cognitive Radio Networks Based on Stochastic Approach
}

\author{
Qiucheng Shan, Dongtang Ma*, Jun Xiong and Xiaoying Zhang \\ College of Electronic Science, National University of Defense Technology, 410073, Changsha, China \\ ${ }^{*}$ Corresponding author
}

\begin{abstract}
Cognitive Radio (CR) is a promising technology for overcoming the lack of available communication bands. However, in Cognitive Radio Networks (CRN), available spectrum changes due to the user mobility. In this paper, we investigate dynamic spectrum planning and allocation in CRN, which considers the mobility of the cognitive users. The on-line dynamic spectrum planning and allocation problem under conventional model is a NP-hard one, which is generally hard to solve due to the unacceptable computational complexity. We propose a model design in a stochastic geometry approach and an off-line dynamic spectrum planning scheme based on this model. Specifically, to evaluate the robustness performance of the cognitive radio networks, we derive the average collision time $T$ in closed form under this model. Further, the value of $T$ will be used to select the best scheme of spectrum utilization. The rationality of this dynamic spectrum planning approach is also verified through computer simulations.
\end{abstract}

Keywords-cognitive radio networks; spectrum planning; stochastic geometry; robustness

\section{INTRODUCTION}

With the rapid development of CR technology, the environmental awareness and the reconfigurable ability of a general hardware platform have greatly ascended, which contribute a lot to the realization of dynamic spectrum planning. Traditional spectrum planning and management technique is a typical kind of static spectrum planning method which licenses a specific radio frequency band to the authorized user based on the command-and-control model [1], without permission, the frequency band could not be changed or occupied by other users. This scheme results in poor efficiency of spectrum utilization, and thus it motivates the dynamic spectrum planning and management in CRN.

There is a comprehensive introduction of dynamic spectrum management and access in CRN in [1], including model designs, planning schemes, performance analysis and access protocols. In conventional method, dynamic spectrum planning is always transformed into a resource allocation problem and further regarded as a constrained optimization problem. Objective functions are in general the throughput of networks, handoff rate and Quality of Service (QoS) of specific terminals etc. In addition, the Constraints mainly include transmission power, spectrum utilization principles and the interference between terminals.

However, the above constrained optimization process is a
NP-Hard problem. The global optimum solution cannot be obtained in accepted complexity with a large enough scale of planning. In addition, when considering the user mobility in highly dynamic networks, the spectrum planning problem becomes more computation consuming even with the heuristic algorithm since the constrained optimization model fails to jump out of the static planning routine. It is hard to fill the gap between the computational complexity and real-time planning requests.

In [2]-[3], Cho's team from KAIST firstly transformed the spectrum planning and allocation into a stochastic geometry problem which solved the probability of collision resulting from the co-channel interference. Specifically, the primary users (PU) were distributed in a spatial Poisson field while secondary users (SU), i.e. CR users (CRU), were supposed to move randomly in a fixed area. When CRU and PU were under the same spectrum, the concept of the "No Talk Zone" was introduced. Once the CRU stepped into the "No Talk Zone" of a PU, it would be deemed that the PU was disturbed by this CRU. However, they did not give the detailed information about the radius of the "No Talk Zone" and the mobility of CRU was unreality.

In [4]-[6], the stochastic geometry has been applied to analyze the performance of wireless networks. However, there are few researches applying the stochastic geometry to the dynamic spectrum planning problem and few solutions of dynamic spectrum planning aiming at dealing with the mobility of CRU have been proposed.

The main contribution of this paper is to propose a model based on stochastic geometry to deal with the dynamic spectrum planning considering the motion of CRU in CRN. In this model, a closed form analytical expression for co-channel collision time $T$ in one motion process is derived through the statistics. By comparing the different collision times under different spectrums, the accessing spectrum can be chosen initiatively. Since the planning basis value $T$ can be off-line computed before the planning decision, the computational complexity of the proposed model is much lower than that of the conventional model based on NP-Hard constrained optimization problem.

\section{Problem Statement AND SyStem ModeL}

The scenario of dynamic spectrum planning with the motion of the CRU is considered in this paper. In a fixed area 
with a central terminal of communication, spectrum resources available must have been used by PUs. When a CRU enters into this area and wants to communicate with the central terminal, which spectrum to access should be addressed. On one hand, CRU should make as little interference as possible to PUs. On the other hand, the communication performance such as the robustness of CRU should not be ignored. In this section, a model is designed to support analyzing the robustness of the networks in a stochastic approach. Based on these analyses, a spectrum planning scheme is also proposed.

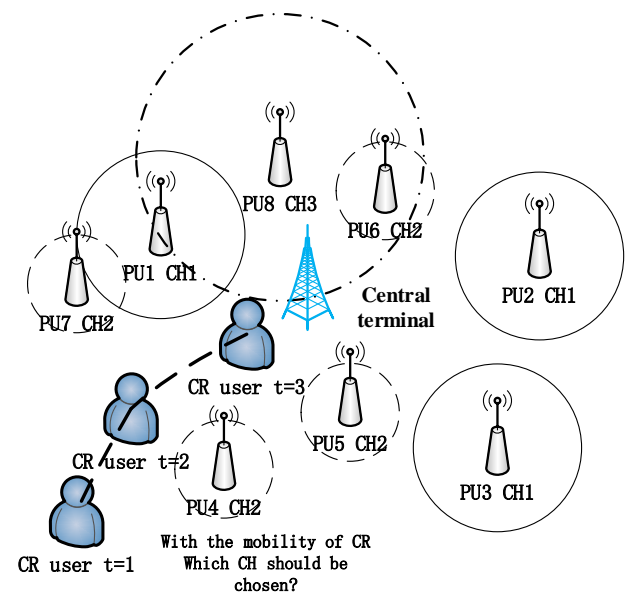

FIGURE I.SCENARIO OF DYNAMIC SPECTRUM PLANNING CONSIDERING THE MOTION OF CRU

As shown in Figure 1. A CRU is moving randomly in a fixed area and wants to communicate with the central terminal. In this area, spectrums available are limited, denoted as $\mathrm{CH}_{i}$ $(i=1,2,3 \cdots N)$, and PUs under different spectrums are located. A concept of "No Talk Zone" is introduced. Once other users' transmitters step into the "No Talk Zone" of a PU under the same spectrum, there will be interference between the two users. Further, the radius of "No Talk Zone" is denoted as $R_{N T}$. In proposed model, the factors which will affect the robustness of the spectrum planning scheme in CRN, such as distance between PUs, the radius of "No Talk Zone" and the motion of CRU should be considered.

\section{A. Distance between PUs under the Same Spectrum}

In stochastic geometry model, Poisson Point Process (PPP) is widely used to describe the scenario in which a fixed number of nodes randomly distributed evenly within a specified range of area. The spatial randomness and independent feature of PPP makes it easy to analyze networks. To deal with the distance between PUs under the same spectrum, we first assume the location distribution of PUs follows PPP.

Denote the distribution of distance to the nth nearest PU as $G_{n}(R)$, we have [7]

$$
G_{n}(R)=1-\exp \left(-\lambda_{i} c_{d} R^{d}\right) \sum_{k=0}^{n-1} \frac{\left(\lambda_{i} c_{d} R^{d}\right)^{k}}{k !}
$$

In (1), $c_{d} R$ is the volume in $d$-dimension space, $\lambda_{i}$ is the Poisson distribution density under the spectrum $i$. When taking the derivative, simplified with Taylor's theorem, all terms in the sum but the one for $n-1$ cancel out, so the PDF is the generalized gamma distribution.

$$
g_{n}(R)=\exp \left(-\lambda_{i} c_{d} R^{d}\right) \frac{\left(\lambda_{i} c_{d} R^{d}\right)^{n} d}{R \Gamma(n)}
$$

Especially, the PDF of the distance between the nearest PUs in two dimensions is

$$
g_{1}(R)=2 \lambda_{i} \pi \operatorname{Re}^{-\lambda_{i} \pi R^{2}} \quad R \in \mathbb{R}^{+}
$$

In conventional PPP, the distance between PUs would vary from 0 to $+\infty$. However, under a specific spectrum, in this practical scenario, especially the radius of "No Talk Zone" $R_{N T}$ is introduced, the distance between PUs would not be smaller than $2 R_{N T}$. If not, there will be interference between PUs under this spectrum. We also assume the range of communication is a circle centered by the central terminal with the radius of $R_{\text {range }}$. In that case, the distance between PUs could not be greater than $2 R_{\text {range }}$. For

$$
\int_{2 R_{N T}}^{2 R_{\text {range }}} g_{1}(R)=\mathrm{e}^{-4 \lambda_{i} \pi R_{N T}{ }^{2}}-\mathrm{e}^{-4 \lambda_{i} \pi R_{\text {range }}{ }^{2}}
$$

Dividing $\mathrm{e}^{-4 \lambda_{\mathrm{i}} \pi R_{N T}{ }^{2}}-\mathrm{e}^{-4 \lambda_{\mathrm{i}} \pi R_{\text {range }}{ }^{2}}$ on both sides, we get

$$
\int_{2 R_{N T}}^{2 R_{\text {range }}} \frac{g_{1}(R)}{\mathrm{e}^{-4 \lambda_{i} \pi R_{N T}{ }^{2}}-\mathrm{e}^{-4 \lambda_{i} \pi R_{\text {range }}{ }^{2}}}=1
$$

Define

$$
f(R)=\frac{g_{1}(R)}{\mathrm{e}^{-4 \lambda_{i} \pi R_{N T}{ }^{2}}-\mathrm{e}^{-4 \lambda_{i} \pi R_{\text {range }^{2}}}} \quad 2 R_{N T} \leq R \leq 2 R_{\text {range }}
$$

Equation (6) is the normalized PDF of distance between PUs in practical scenario.

\section{B. Radius of "No Talk Zone"}

We assume that except for the central terminal and CRU, the transmit powers of all the PUs are the same. So, under different spectrums, the radiuses of "No Talk Zone" are also different for different path loss under different spectrums. Under a specific spectrum $i$, the communication range of the central terminal is assumed as $R_{\text {range }}$, a CRU is transmitting messages to the central terminal. At the receiver of a PU, the interference comes not only from the other PUs, but also from the CRU. The worst case of the PU's down-link communication is mainly taken into consideration as shown in 
Figure 2. A PU is located at the communication boundary of the central terminal under spectrum $i$ for the minimum receiving power from the central terminal and the distance between the PU and the central terminal is $R_{\text {range }}$. Meanwhile, the CRU is also at the boundary of this PU's "No Talk Zone" for the maximum interference power from the CRU. The distance between CRU and the PU is $R_{N T}$. All other PUs using the same spectrum are transmitting messages for the maximum interference power from the other PUs.

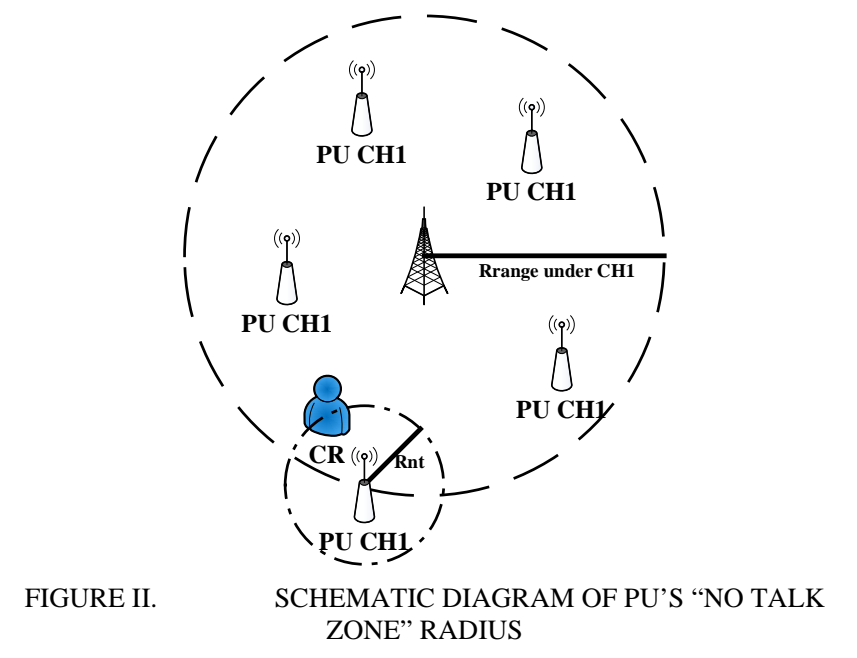

The signal to interference plus noise ratio (SINR) at the PU's receiver under consideration in this scenario is:

$$
\operatorname{SINR}=\frac{P_{C}\|h\|^{2} R_{\text {range }}{ }^{-\alpha}}{P_{C R}\|h\|^{2} R_{N T}^{-\alpha}+W+\mathrm{EI}}
$$

In (7), the transmitting power of central terminal $P_{C}$, the transmitting power of the CR user $P_{C R}$, the power of noise $W$ and the channel gain $h$ are known to us. Under the specific spectrum $i$, the radius of communication area $R_{\text {range }}$ could be calculated. We assume the threshold of PUs' receiver is $\gamma_{\text {thre }}$. We can obtain the $R_{N T}$ with $R_{\text {range }}$ under spectrum $i$ through the equalization $\operatorname{SINR}=\gamma_{\text {thre }}$ if the average accumulated interference from the other PUs, EI can be derived.

Under the given spectrum $i$, as mentioned in section $\mathrm{A}$, the locations of PUs follow the PPP with intensity of $\lambda_{i}$. For the homogeneity of PPP, we can assume the PU under consideration is at the origin. The accumulated interference from all other PUs can be denoted as:

$$
I=I(o) \stackrel{\Perp}{=} \sum_{x \in \Phi} P h_{x} l(x)=\sum_{x \in \Phi} P h_{x}\|x\|^{-\alpha}
$$

In (8), the point process $\Phi$ is PPP, $h_{x}$ is the channel gain while the $x$ is the distance between the other PUs to the origin, the transmitting power of PUs is $P$. The path loss law is typically of the form $l(x)=\|x\|^{-\alpha}$. To simplify the model, we assume the $P$ and $h_{x}$ are normalized to 1 . For the Campbell's formula:

$$
\mathrm{E} I=\lambda_{i} \int_{R^{d}}\|x\|^{-\alpha} d x
$$

In two dimensions,

$$
\begin{aligned}
\mathrm{EI} & =\lambda_{i} \int_{R^{d}}\|x\|^{-\alpha} d x=\lambda_{i} \int_{0}^{2 \pi} \int_{0}^{\infty} r^{-\alpha} r d r d \varphi \\
& =\left\{\begin{array}{l}
\left.2 \pi \frac{\lambda_{i}}{2-\alpha} r^{2-\alpha}\right|_{0} ^{\infty} \\
2 \pi \lambda_{i} \ln r \mid \begin{array}{l}
\infty \\
0
\end{array}
\end{array}\right.
\end{aligned}
$$

As mentioned in section $\mathrm{A}$, the distance between the PUs is no smaller than $2 R_{N T}$ and no greater than $2 R_{\text {range }}$ under the given spectrum $i$. So, (10) should be changed into:

$$
\mathrm{E} I=\left\{\begin{array}{l}
2 \pi \frac{\lambda_{i}}{2-\alpha}\left[\left(2 R_{\text {range }}\right)^{2-\alpha}-\left(2 R_{N T}\right)^{2-\alpha}\right], \alpha \neq 2 \\
2 \pi \lambda_{i} \ln r \mid \begin{array}{l}
2 R_{\text {range }} \\
2 R_{N T}
\end{array}=2 \pi \lambda_{i} \ln \left(\frac{R_{\text {range }}}{R_{N T}}\right), \alpha=2
\end{array}\right.
$$

With the value of EI, we can calculate the $R_{N T}$ under a specific spectrum $i$.

\section{Motion Model of CRU}

In practical scenario, a mobile CRU always chooses a destination in communication area, moves to it and then pauses for a period of time. Then, it will move to the next destination. Considering the practical movements in this scenario, we choose the Random WayPoint (RWP) to describe the motion of the CRU.

In RWP, the destination and speed are chosen randomly and independently at any step of the model. The CRU begins at the source point $\mathrm{S}$ which is selected randomly in the communication area. Then the CRU will select another destination point $\mathrm{D}$ randomly and move to it at a random speed $v$ between $v_{\min }$ and $v_{\max }$. At point $\mathrm{D}$, the CRU will pause for a period of time denoted as $t_{\text {pause }}$ which varies uniformly from $t_{\min }$ to $t_{\max }$. It is a step of the RWP and after a step, the previous $\mathrm{D}$ becomes $\mathrm{S}$ in the next step. The behavior is repeated for a given time.

\section{Proposed Planning Scheme}

In this section, we introduce the average collision time $T$ to evaluate the robustness performance of CRN. $T$ represents the average time that the CRU stays in the "No Talk Zone" of a PU under a specific spectrum in each step of RWP. The closed 
expression of $T$ is addressed and we can decide which spectrum to access depending on the value of the $T$.

Specifically, under spectrum $i$, there must be a PU B nearest to the PU A. In the model of RWP, the motion range of CR user is denoted as $R_{m}$, in order to prevent the interference to the PU B, $R_{m}=R-R_{N T}$. $R$ is the distance between $\mathrm{A}$ and $\mathrm{B}$ under PPP model, $R_{N T}$ is the radius of "No Talk Zone".

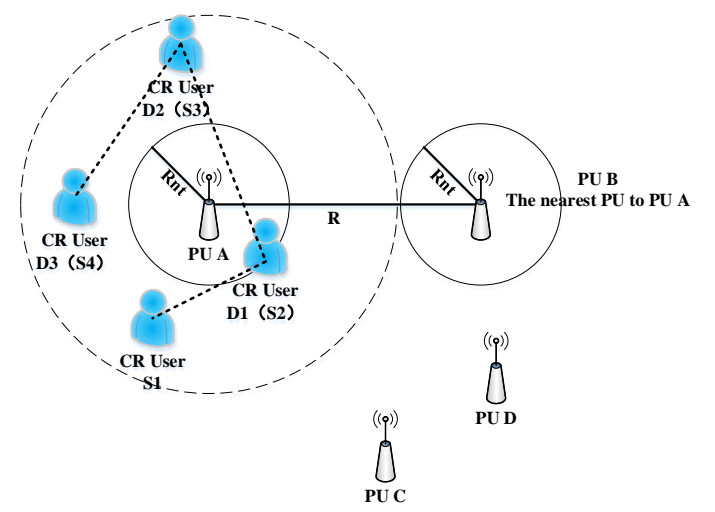

FIGURE III. RANGE OF MOTION AREA AND COLLISION TIME UNDERSTANDING UNDER RWP

The circle centered by PU A with the radius of $R_{N T}$ is denoted as $\operatorname{Reg}\left(R_{N T}\right)$. By the same analogy, the $\operatorname{Reg}\left(R_{m}\right)$ is also defined. As displayed in figure 3 , since the source and destination point distribute randomly and independently in a finite area. There are 5 possible cases:

(1) S,D are outside the "No Talk Zone", and SD cuts the $\operatorname{Reg}\left(R_{N T}\right)$;

(2) S,D are outside the "No Talk Zone", and SD does not cut the $\operatorname{Reg}\left(R_{N T}\right)$;

(3) S,D are inside the "No Talk Zone", and SD is inside the $\operatorname{Reg}\left(R_{N T}\right)$;

(4) One of S and D is inside the "No Talk Zone", with the other outside, and SD cuts the $\operatorname{Reg}\left(R_{N T}\right)$;

(5) One or two of $S$ and D is at the boundary of $\operatorname{Reg}\left(R_{N T}\right)$ or $\operatorname{Reg}\left(R_{m}\right)$
To sum up, in case (2), the CR user does not step into the "No Talk Zone" and the probability of case (5) is zero, so both of them are ignored. Indeed, there are 3 cases to be considered.

Under the model of RWP, the number of the CRU's steps is $n$. The total time that the CRU stays in the "No Talk Zone" is $T_{N T}=T_{\text {NTPause }}+T_{\text {NTMove }} . T_{\text {NTPause }}$ is the total pause time in "No Talk Zone" and $T_{\text {NTMove }}$ is the movement time in "No Talk Zone”. It is easy to know:

$$
T_{\text {NTPause }}=\frac{1}{2}\left(P_{\text {inout }}\left(R_{N T}\right) n \bar{t}+2 P_{\text {inin }}\left(R_{N T}\right) n \bar{t}\right)
$$

In (12), $P_{\text {inout }}$ is the probability of case (4) while $P_{\text {inin }}$ is the probability of case (3), $\bar{t}$ is the average pausing time at a point and $\bar{t}=\frac{t_{\min }+t_{\max }}{2}$.Obviously

$$
T_{\text {NTMove }}=n \frac{\overline{d\left(R_{N T}\right)}}{\bar{v}}
$$

In (13), $n \overline{d\left(R_{N T}\right)}$ is the total path length in "No Talk Zone" within $n$ steps, $\bar{v}$ is the average velocity and $\bar{v}=\left(v_{\max }-v_{\min }\right) / \ln \frac{v_{\max }}{v_{\min }}$. The derivation of $\bar{v}$ was given in [8]. So the average path length in "No Talk Zone" in each step can be obtained. When $n \rightarrow \infty$

$$
\begin{gathered}
\overline{d\left(R_{N T}\right)}=P_{\text {inin }}\left(R_{N T}\right) \cdot \overline{d_{\text {inin }}\left(R_{N T}\right)}+P_{\text {outout }}\left(R_{N T}\right) \cdot \\
\overline{d_{\text {outout }}\left(R_{N T}\right)}+P_{\text {inout }}\left(R_{N T}\right) \cdot \overline{d_{\text {inout }}\left(R_{N T}\right)}
\end{gathered}
$$

According to the mathematical conclusion, the probability of random dotting in concentric circles and average length in inner circle of random strings is easily known:

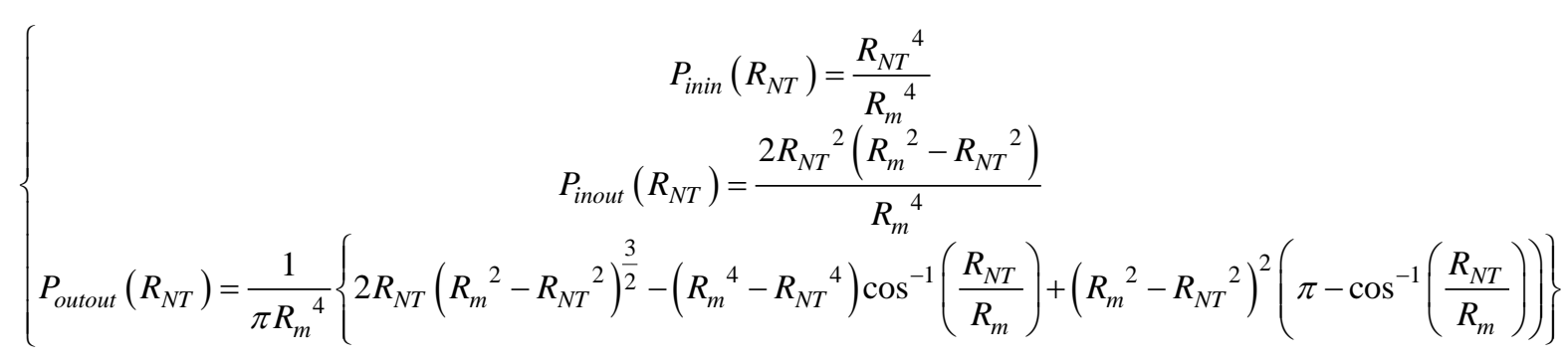




$$
\begin{aligned}
& \left\{\overline{d_{\text {outout }}\left(R_{N T}\right)}=\frac{\overline{d_{\text {inin }}\left(R_{N T}\right)}=\frac{128}{45 \pi} R_{N T}}{\left\{\frac{1}{4} \pi\left(R_{m}{ }^{2}-R_{N T}{ }^{2}\right)-\frac{1}{2} R_{m}{ }^{2} \cos ^{-1}\left(\frac{R_{N T}}{R_{m}}\right)+\frac{1}{2} R_{N T} \sqrt{R_{m}{ }^{2}-R_{N T}^{2}}\right.}\left\{\int_{0}^{R_{N T}} 2\left(\sqrt{\left(R_{m}{ }^{2}-\rho^{2}\right)\left(R_{N T}{ }^{2}-\rho^{2}\right)}-\left(R_{N T}{ }^{2}-\rho^{2}\right)\right) d \rho\right\}\right. \\
& \overline{d_{\text {inout }}\left(R_{N T}\right)}=\frac{8}{3 \pi R_{N T}{ }^{2}\left(R_{m}{ }^{2}-R_{N T}^{2}\right)} \int_{R_{N T}}^{R_{m}} \int_{0}^{\sin ^{-1}\left(\frac{R_{N T}}{\xi}\right)}\left(R_{N T}^{2}-(\xi \sin \theta)^{2}\right)^{\frac{3}{2}} \xi d \theta d \xi+\frac{8 R_{N T}}{3 \pi}
\end{aligned}
$$

In RWP, the average time in "No Talk Zone" in each step $\overline{t_{\text {step }}}=T_{N T} / n$. Under a spectrum $i$, since $R_{N T}$ and $R_{\text {range }}$ are given, $\overline{t_{\text {step }}}$ is the function of $R_{m}$. As $R_{m}=R-R_{N T}, \overline{t_{\text {step }}}$ is the function of $R$ of which the PDF is deduced in (6) under model PPP.

So under the given spectrum $i$, the average time is $T$ and

$$
T=\int_{R_{N T}}^{2 R_{\text {range }}-R_{N T}} \overline{t_{\text {step }}} f\left(R_{m}\right) d R_{m}=\int_{2 R_{N T}}^{2 R_{\text {range }} \overline{t_{\text {step }}}} f(R) d R(17)
$$

\section{SimUlation RESUlts AND DisCUSSION}

In this section, the proposed frequency planning scheme is assessed through simulation. We consider the movement of CR user in concentric circles depicted in Fig 3. The outer circle represents the range of motion in model RWP and the inner circle represents the "No Talk Zone". In a given simulation time, the number of steps and the total time in "No Talk Zone" are accumulated. If the simulation time is long enough, the quotient of the calculated total time in "No Talk Zone" and number of RWP steps is convergent to the average time in "No Talk Zone" in each step. By comparing the value of $\overline{t_{\text {step }}}$ from simulation and theoretical analysis, we can analyze the accuracy of proposed model.

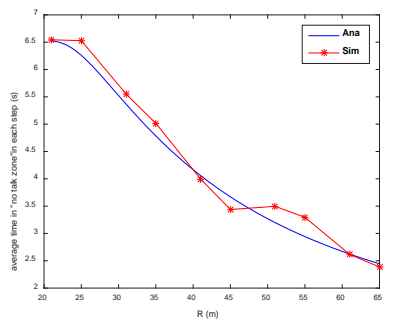

(a)

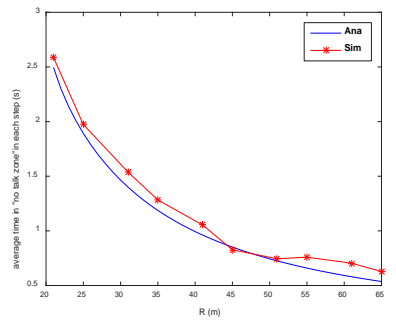

(c)

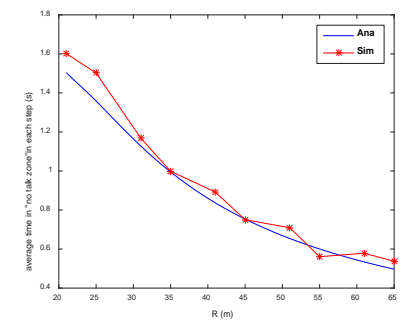

(b)

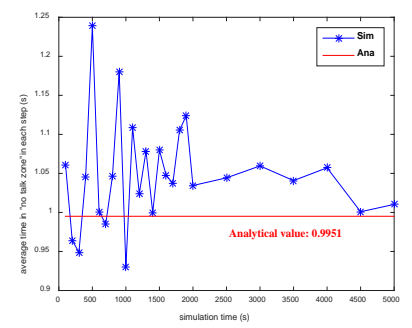

(d)
FIGURE IV. SIMULATION RESULTS VS. ANALYTICAL RESULTS UNDER DIFFERENT CONDITIONS AND ASTRINGENCY OF SIMULATION (a) $\quad t_{\text {pause }} \in[0.1,0.5](\mathrm{s}) \quad, \quad v \in[1,2](\mathrm{m} / \mathrm{s}) \quad, \quad R_{N T}=10 \mathrm{~m} \quad$; (b) $t_{\text {pause }} \in[0.1,0.5](\mathrm{s}), \quad v \in[5,10](\mathrm{m} / \mathrm{s}), R_{N T}=10 \mathrm{~m}$; (c) $t_{\text {pause }} \in[1,2](\mathrm{s})$, $v \in[5,10](\mathrm{m} / \mathrm{s}) \quad, \quad R_{N T}=10 \mathrm{~m} \quad ; \quad$ (d) astringency of simulation $t_{\text {pause }} \in[0.1,0.5](\mathrm{s}), v \in[5,10](\mathrm{m} / \mathrm{s}), R=35 \mathrm{~m}, R_{N T}=10 \mathrm{~m}$

In the figure 4, especially in (a)(b)(c), under different ranges of $t_{\text {pause }}$ and $v$, the downward trends of the simulation results (Sim) and the analytical results (Ana) are almost the same with the increase of $R$. The rationality of the $\overline{t_{\text {step }}}$ from theoretical analysis under the proposed model has been proved. However, we cannot ignore the difference between the convergence value from simulations (Sim) and the analytical value (Ana) in (d). The deep reason is that the RWP model is, generally non-uniform. Although the selection of $\mathrm{S}$ and $\mathrm{D}$ follows a uniform random distribution, the mobility model changes this distribution during the simulation. It occurs because nodes tend to cross the center of the motion area with a relatively high frequency, which is proved in [9]. So the result of the simulation is a little larger than the analytical result which is derived under the uniform distribution of $\mathrm{S}$ and D.

However, the analytical average time in "No Talk Zone" $T$ in our proposed model is used to distinguish the robustness under different spectrums. In other words, as long as it can be used to distinguish the different performance under different spectrums, some deviations in absolute value can be accepted.

\section{CONCLUSION}

In this proposed model of dynamic spectrum planning which considers the motion of the CRU, we succeed in applying the stochastic geometry into the spectrum planning and allocation problem. Further, we calculate the theoretical average time $T$ in "No Talk Zone" which contains all the influence factors when we consider the robustness of CRN under proposed model. Based on that, a spectrum utilization scheme is also proposed before the CRU intends to access the network. The computational complexity is greatly reduced and the dynamic spectrum utilization is improved. To some extent, dynamic spectrum planning under this model is a kind of initiative planning.

\section{ACKNOWLEDGEMENTS}

This research was supported by the Natural Science Foundation of China, No.61372099, 61601480.

\section{REFERENCES}

[1] Ekram Hossain, "Dynamic Spectrum Access and Management in Cognitive Radio Networks”: Cambridge University Press, 2009. 
[2] W. Lee and D. H. Cho, "Channel Selection and Spectrum Availability Check Scheme for Cognitive Radio Systems Considering User Mobility”, IEEE Commun. Lett., vol. 17, no. 3, March, 2013.

[3] W. Lee, Robert Schober, "Analysis of Coverage in Heterogeneous Cellular Networks,” IEEE Commun. Lett., vol. 20, no. 6, June, 2016.

[4] A. Ijaz, S. A. Hassan, S. A. R. Zaidi, D. N. K. Jayakody and S. M. H. Zaidi, "Coverage and Rate Analysis for Downlink HetNets Using Modified Reverse Frequency Allocation Scheme”, IEEE Access, vol.5 pp.2489-2502, March, 2017.

[5] K.Cho J. Lee, Chung G. Kang, "Stochastic Geometry-Based Coverage and Rate Analysis under Nakagami Log-normal Composite Fading Channel for Downlink Cellular Networks,” IEEE Commun. Lett., vol. 21, no. 6, pp. 1437-1440,2017.

[6] T. Ohto, K. Yamamoto, Seong Lyun Kim, T. Nishio., "Stochastic Geometry Analysis of Normalized SNR-Based Scheduling in Downlink Cellular Networks,” IEEE Wirel. Commun. Lett., vol. 6, no. 4, pp. 438441, 2017

[7] Martin Haenggi, "Stochastic Geometry for Wireless Networks," Cambridge University Press, 2012.

[8] Shi Rui and Yang Xiaozong, "Research on the Node Spatial Probability Distribution of the Random Waypoint Mobility Model for Ad Hoc Network", Journal of Computer Research and Development, Vol.42 No.12, pp.2056-2062, 2005.

[9] Bettetetter C, Resta G, Santi P. "The Node Distribution of The Random Waypoint Mobility Model for Wireless Ad-Hoc Networks," IEEE Transactions on Mobile Computing, Vol.2 No.3 pp.257-269, 2003. 